\title{
LA EVALUACIÓN DE LOS GERENTES PÚBLICOS FRENTE A LA OPINIÓN PÚBLICA
}

\author{
ANABELLE CASTILLO LÓPEZ \\ Escuela de Ciencias de la Administración \\ Universidad Estala a Distancia, Costa Rica \\ acastillo@uned.ac.cr
}

\section{RESUMEN}

A nivel mundial, la crítica al sector público -que tiene mucho de verdad-, está alcanzando niveles de tal magnitud que ha llevado a los ciudadanos a una enorme pérdida de confianza en dicho sector, tanto así que han sido fácilmente convencidos de que los asuntos públicos deben y pueden resolverse fuera de la institucionalidad de dicho sector. Dichas críticas han conducido también a académicos a proponer cambios en la organización y funcionamiento del sector público. El presente trabajo parte del hecho de que toda esa crítica debe ser conducida a la luz de criterios objetivos, del conocimiento profundo sobre el funcionamiento propio de este sector y tomando en cuenta las características que lo distinguen del sector privado. Se parte aquí de la premisa de que el sector público tiene una serie de complejidades que lo hacen diferente al sector privado y que no se pueden resolver fácilmente, puesto que la cobertura de su funcionamiento requiere llegar a todos los ciudadanos de un país con productos y servicios de alta dificultad, lo que lo obliga a diseñary poner en funcionamiento sistemas administrativos muy complejos para la prestación de los servicios; siendo ello diferente al sector privado, que tiene mercados cautivos, para productos muy bien delimitados. Desde esta perspectiva, tampoco es suficiente con trasladar la producción de bienes y servicios al sector privado, puesto que estaríamos trasladando todas esas complejidades a ese sector, con lo cual caeríamos en la misma situación, solo que en un sector diferente. Por tal razón, este análisis trata de aportar sobre cuáles son esas características propias del sector público que debemos tomar en cuenta a la hora de evaluarlo y sobre cuáles son parámetros que sí podríamos utilizar para llevar a cabo una evaluación objetiva de las actuaciones del sector público.

PALABRAS CLAVE: GERENCIA PÚBLICA, SECTOR PÚBLICO, EVALUACIÓN, OPINIÓN PÚBLICA, EFICIENCIA, EFICACIA.

\section{ABSTRACT}

Public sector mal-functioning has long been criticized and, as a result, great disappointment and a loss of confidence have grown among citizens who are now convinced that public activities should be transferred into the private sector. Because of that, academics all over have dedicated time to study this phenomenon and suggested new academic approaches, many of which are oriented to the adoption of alternative methods to manage public organizations as private enterprises or to definitively transfer public activities to private sectors. Therefore, this work tries to point out that, in order to give appropriate judgments or assessments about the public sector, it is necessary to clarify certain complexities that characterize it. These clarifications are important because misjudgments are usually expressed by different evaluators -journalists, auditors, etc. - through the media. That ends up in misunderstandings and a lack of support to public affairs. Clarifications are also necessary not only because people deserve unbiased assessments of the public sector, but also because, probably for them, it is not easy to recognize what is good and what is wrong with the public sector. Moreover, this work shows that there are no easy ways to go around many public affairs because, even though some activities can be transferred to the private sector, not all of them can, and, at the end, due to specific comple- 
xities, we will be simply transferring those complexities from one sector to another keeping the problems intact. It is recognizable that, in the public sector, things can be improved, but expectations over performance should be in accordance with reality. Finally, this study shows some parameters to positively measure public activities in order to monitor and contribute to better governance.

KEYWORDS: PUBLIC MANAGEMENT, PUBLIC SECTOR, EVALUATION, PUBLIC OPINION, EFFICIENCY, EFFECTIVENESS.

\section{INTRODUCCIÓN}

Existe una apreciación negativa generalizada a nivel mundial sobre el sector público, que tienen mucho de verdad, pero que ha conducido a académicos, analistas y comentaristas a un nivel de criticismo tal, que hasta hacen creer a la opinión pública que los asuntos de este sector se pueden y se deben resolver fuera de la institucionalidad de este. Prueba de ello es el convencimiento de que los servicios que tradicionalmente habían estado en manos del sector público pueden y deben ser trasladados al sector privado, tal es el caso de la administración del agua, aeropuertos, carreteras, educación, salud y otros, mediante la creación de leyes que permiten la concesión, o venta de tales servicios (Al respecto: Zurcher, 2014; Molina y Chowla, 2008).

Como resultado se han planteado una serie de debates entre académicos y especialistas para cambiar el rumbo de la gestión pública y lograr un mejor gobierno.

Producto de ese trabajo de análisis tenemos una serie de enfoques que van desde temas sobre cómo introducir la gestión de la calidad, la reingeniería y otros en la administración pública, tal y como lo establecen los planteamientos en los enfoques de la Nueva Gerencia Pública (Olías de Lima, 2001; Schroeder, 2009), hasta cómo retomar conceptos muy propios de la administración pública como la descentralización y la descon- centración, para cambiarlos por una propuesta de desregulación y traslado a manos privadas en sectores como educación, salud, electricidad y otros. Ejemplo de esto es la Ley de Creación del CONESUP de 1981 y la creación de cooperativas de salud con uso de infraestructura de la Caja Costarricense de Seguro Social (Maingón, 1991). Todo lo anterior con el fin de lograr una mayor eficiencia y alcanzar eficacia, bajo métodos de trabajo que buscan lograr que la administración pública funcione lo más cercano posible a una empresa privada.

Sin embargo, si bien la intención inicial de ello es lograr mejoras en el funcionamiento del Sector Público, lo que sí es cierto es que en muchos casos han traído confusión y desconcierto a administradores y gerentes de estas instituciones, tal es el caso de las dificultades que se presentan a la hora de supervisar correctamente la prestación de los estos servicios (véase los señalamientos que se hacen a la supervisión de los ingresos de Autopistas del Sol, Periódico Digital CRHOY, 9 de octubre 2013).

Además, la aplicación de tales principios, tampoco ha sido lo que se esperaba. Es así como en el caso de Costa Rica, conviven intentos de dar una mayor flexibilidad al sector público por medio de la contratación privada de servicios, con un marco legal que cada vez reduce más las posibilidades de actuación de la gerencia pública, por lo que dificulta las posibilidades de lograr un nivel razonable de eficiencia y eficacia en este sector.

Lo cierto es que frente a todos esos cambios y los resultados que se están obteniendo en cuanto a calidad y cantidad de bienes y servicios del sector público, se requieren efectivamente acciones que conduzcan a someter a gerentes e instituciones a evaluaciones que lleven a determinar si los resultados de tales innovaciones son los adecuados, en términos de costos, eficacia y 
beneficios a la ciudadanía, siendo este el tema que nos ocupa en este análisis.

Pero, si bien la evaluación y los evaluadores son necesarios, tratándose del Sector Público, por lo complejo del tema, se requiere un profundo estudio del mismo para que las apreciaciones que se realizan sean objetivas y cumplan un papel constructivo frente a la opinión pública, es decir que contribuyan a desarrollar una Administración Pública lo más cercana posible a los ciudadanos a los que sirve, a informar a los administrados con criterios objetivos sobre el verdadero funcionamiento del sector, a advertir sobre el verdadero costo de sus actividades y propiciar que dichas actividades sean lo más transparentes posible.

\section{Metodología de análisis}

Frente a esa problemática, se hace necesario entonces someter a análisis las consideraciones que deben tenerse a la hora de realizar evaluaciones sobre el Sector Público a fin de convertirlas en instrumentos de mejora de sus propias actuaciones.

Se parte aquí de la premisa de que tales evaluaciones deben ser tanto el apoyo que permita a los encargados tomar mejores decisiones, orientarse y avanzar en la ejecución de los programas nacionales, como el instrumento que permita a la ciudadanía contar con elementos de juicio para sopesar técnicamente la eficiencia y eficacia de este sector y diferenciarlos de los intereses de la política.

Aunque es de sobra conocido en teoría administrativa, que la evaluación es un recurso fundamental para detectar problemas de eficacia -evaluación de impacto-, de eficiencia -evaluación de rendimiento- y de gestión del programa -evaluación de la implantación (Bañón 1997)-, las actuaciones del sector público tienen sus características particulares que deben ser to- madas en cuenta a la hora de realizar cualquier valoración. Este análisis, por lo tanto, pretende reflexionar sobre los errores en que se puede caer al precipitar juicios de valor con valoraciones que van a tener trascendental importancia en la opinión de los ciudadanos.

Por esta razón, se trata aquí de hacer un aporte sobre algunos criterios importantes a tomar en cuenta a la hora de juzgar la actuación del Sector Público, sobre la forma en que se analizan sus actuaciones, sobre la necesidad de tomar en cuenta el contexto en el que se desarrolla y sobre las complejidades que le son propias y que no permiten compararlo con ningún otro sector.

El presente trabajo no intenta justificar actuaciones erróneas de la Administración Pública, sino anotar ciertas consideraciones importantes que deben ser tomadas en cuenta en la actividad evaluativa, precisamente para obligar a dicho sector a actuar de una mejor forma.

Se pretende hacer una reflexión fundamentada en bibliografía, observaciones y experiencias personales sobre el caso particular de Costa Rica, pero es probable que también aplique para otros países.

La importancia de este estudio se basa en el hecho de que los juicios emitidos sobre el Sector Público -equivocados o no-, marcan derroteros por los cuales los gerentes públicos conducen al país, puesto que la presión que se ejerce sobre ellos por parte de la opinión pública es finalmente un elemento importante a considerar en la toma de decisiones. Se forma así un círculo donde la retroalimentación que el gerente recibe de la opinión pública influye en las decisiones que toma, y por lo tanto, ir en contra de la opinión pública podría significar la pérdida de poder, de funciones o ambas por parte de un funcionario. 


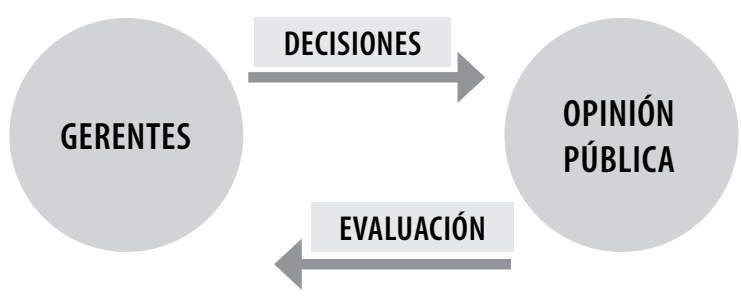

Fuente: elaboración propia, 2013.

Más aún, la percepción, el apoyo y las respuestas de los ciudadanos a las actuaciones de la administración pública pueden depender de la información que ellos reciben sobre los resultados de esta.

Para los efectos de este análisis, se entiende por evaluación los juicios, opiniones o valoraciones que se hacen a partir de resultados de estudios sobre actuaciones del sector público, de interpretaciones que se realizan sobre su eficiencia y eficacia, a partir de la observación, entre otros.

No se trata aquí de analizar evaluaciones realizadas, sino de examinar elementos del contexto del sector público que deben ser tomados en cuenta para colocar tales juicios en su justa dimensión.

Si bien desde el punto de vista de la teoría administrativa se entiende por gerente todo aquel que tiene personal a cargo (Robbins y Coulter 2011), para el presente análisis se entenderá como gerente público a los más altos dirigentes de las instituciones públicas, es decir, aquellos de alto nivel que ocupan cargos de ministros, viceministros y directores en instituciones públicas, tanto del gobierno central como descentralizado. No se hará mención de los gerentes de menor nivel puesto que las evaluaciones que se hacen de ellos generalmente no están en el centro del debate público -salvo algunas excepciones- y por lo tanto, no hay mayores juicios de valor que influyan de manera determinante en su actuación ni en las políticas que la institución asume como consecuencia de los mismos, aunque su trabajo sí es importante para la consecución de los resultados en las instituciones.

Es importante aclarar que en Costa Rica se han hecho grandes esfuerzos dirigidos a efectuar evaluaciones sobre los resultados de las instituciones públicas en el mejoramiento en la calidad de vida y en áreas como la educación y la salud. Sin embargo, el objetivo de este trabajo es realizar un análisis de las consideraciones que se hacen con respecto al gerente público y a la medición de lo que él propone como resultados. Se trata entonces de un aspecto muy particular de la Administración Pública (el gerente y su entorno) que podría ayudar a comprender mejor las derivaciones de cualquier evaluación.

Por lo tanto, con el objeto de analizar dichas apreciaciones públicas y sus consecuencias, se comenzará entonces por describir cuáles son los principales evaluadores que aportan criterios a la opinión pública, para luego arribar en algunas complejidades del contexto en que se desarrolla un gerente público, y posteriormente establecer ciertos criterios con los que se podrían evaluar dichos funcionarios. Finalmente el análisis considera algunas consecuencias de evaluaciones erróneas que enfrentan a gerentes e instituciones públicas con la opinión pública.

\section{El papel de los evaluadores}

No cabe duda de que el papel de los evaluadores es de gran importancia, y es por esta razón que han surgido gran cantidad de ellos como la prensa, instituciones académicas, Contraloría General de la República y otros auditores. Surgen así actores importantes por su influencia en la opinión pública y en la forma en que se conduce la Administración Pública.

En el caso costarricense la prensa constituye uno, y quizá el más importante, protagonista, puesto que es el que más contribuye a formar 
opinión en los costarricenses. Lo anterior se comprueba en la Primera Encuesta Nacional de Hábitos y Prácticas Culturales, Costa Rica 20102011 (llevada a cabo por la Dirección de Cultura del Ministerio de Cultura, Juventud y Deportes de Costa Rica), donde se demuestra que el $51 \%$ de los costarricenses no leyó ningún libro en el último año, pero cerca del $76 \%$ de la población lee el periódico. De la misma manera, ocho de cada 10 personas de mayores de 6 años (83.4\%) indicaron que acostumbran a oír radio, con un promedio nacional de cerca de 4 horas (Ministerio de Cultura, 2010).

Más aun, según esta misma encuesta, la televisión es el medio de comunicación más utilizado por la población donde el 98,2\% de las personas mayores de 6 años manifestaron que acostumbran ver televisión y lo hace como promedio de 4 horas diarias (Ministerio de Cultura, 2010).

Aunque no se puede deducir de esta encuesta cuánta es la influencia de los medios de comunicación en la opinión pública en general, sí se tiene evidencia en ámbitos específicos sobre tal influencia. Por ejemplo, un estudio realizado por el Instituto de Estudios Comparados en Ciencias Penales y Sociales (INECIP) denominado "Monitoreo Anual sobre Independencia Judicial y Asociacionismo en Costa Rica" verificó que entre enero de 2002 y febrero de 2003 se realizó una encuesta a funcionarios judiciales donde se establece que dentro de los grupos que ejercen mayor presión a los diversos funcionarios y funcionarias del Poder Judicial, en primer lugar se encuentra la prensa (Vargas, 2008, citado por Porter, 2010).

En el caso de la Contraloría General de la República, esta asume cada vez más el rol de evaluador y que representa otro de los grandes actores que forman opinión en la población costarricense. Según declaraciones de la Contralora General de la República los controles que utiliza este ente deben prevenir, detectar, corregir, las de- bilidades y riesgos de las distintas instituciones públicas, de manera que quede claro su papel evaluador (Acosta, 2012).

Lo importante de tomar en consideración es que como los juicios de todos estos importantes evaluadores se hacen a nivel mediático, de nuevo la prensa cobra enorme importancia al ser el filtro por medio del cual el ciudadano recibe la información sobre la mayoría de evaluaciones del sector público, convirtiéndose en el medio más importante para formar opinión.

\section{Complejidades para evaluar el cumplimiento del gerente público}

En este apartado se hará un análisis de características propias de la administración pública que hacen difícil su estudio. Se planteará una serie de complejidades del contexto de este sector que dificultan el logro de la eficiencia y eficacia y que condicionan su actuación, por lo que deben ser tomados en cuenta en las evaluaciones.

No se pretende con ello justificar las ineficiencias del sector público, ni tampoco hacer ver a los gerentes como víctimas de las complejidades de su entorno, puesto que un gerente debe saber trabajar con esos obstáculos, pero tampoco se pueden ignorar porque se caería en una visión miope y parcializada que no puede ver tales diferencias y por lo tanto, puede tender a igualar las circunstancias del Sector Público con las circunstancias propias de las empresas privadas.

Dentro de las características propias del Sector Público que deben tenerse en consideración al entrar a juzgar su actuación tenemos las que se presentan a continuación, sin tratar de ser exhaustivo.

En primer lugar, es necesario tener presente, que los programas del sector público son difíciles de llevar a cabo y toman tiempo. A diferencia del sector privado, aquí no existen mercados delimi- 
tados sino una cobertura a nivel nacional que requiere una extensa labor de planificación, organización y dirección a partir de lo cual se pueden comenzar a observar resultados (Castillo, 2012).

Es así como el gerente público necesita tiempo para el análisis, reflexión y ejecución, es decir, toda acción debe ser cuidadosamente planeada, aun cuando la planificación puede llevarse a cabo de muchas maneras. Dependiendo de la actividad que se trate, la planificación puede ser el resultado de un documento cuidadosamente elaborado y consultado con todos los involucrados, puede estar apoyada en una serie de estudios y datos; puede ser solo una serie de ideas y procedimientos sobre la forma en que se deben mejorar las acciones para continuar la ejecución de un programa existente, o bien, pueden ser estudios de factibilidad para la apertura de un nuevo proyecto. Lo cierto es que el camino a seguir debe estar muy claro en la mente de quien conduce el programa: el gerente público (CastiIlo, 2012).

En segundo lugar, una vez planificado, emprender un nuevo proyecto es una labor compleja tanto en el sector público como en el privado. En el primero es muy probable que dichas complejidades aumenten. En la administración pública interactúan una serie de valores, normas, motivaciones políticas y comportamientos que son los que finalmente conducen a decisiones. Efectivamente, aquí existe un cuerpo normativo que es más complicado que las reglas que rigen una empresa privada. Se presenta también una cadena de motivaciones y valores de tipo político que son confusos, unos valores democráticos que se deben cumplir tales como el servicio al bien común y hasta una contratación de recursos basada en complejos sistemas de selección, todo lo cual crea una serie de conflictos y contradicciones entre ellos, que dificulta la toma de decisiones (Pullit, 2003,). Además, la gran cantidad de negociaciones que se requiere para emprender un programa en el sector público, el número de actores que interviene y los procedimientos que exige el marco legal marcan una enorme diferencia con el sector privado.

Es así como las tareas a nivel nacional del sector público no pueden realizarse en el mismo tiempo en que se realizan en el privado.

En tercer lugar, tenemos algunas características a considerar para realizar cualquier evaluación al sector público. Por ejemplo, la lectura de ciertas cifras sobre la ejecución hecha en ambientes tan complejos como el que se desenvuelven las instituciones públicas, podría llevar a conclusiones erróneas. De esta manera, no se debería comparar un municipio que tiene algunos elementos de alta calidad como sería contar con buenos profesionales y tecnología moderna, con otro donde no existen los mismos elementos de calidad porque no cuenta con recursos para adquirirlos. De esta manera, si se comparan ambos municipios, midiendo sus resultados sin tomar en cuenta las anteriores consideraciones, se llegaría a conclusiones erróneas sobre su funcionamiento.

De la misma manera, podrían realizarse comparaciones entre programas similares llevados a cabo en diferentes lugares, para lo cual debe tenerse el cuidado de eliminar las discrepancias, producto de los diferentes contextos en que se logran los resultados.

Se hace necesario entonces entender los distintos factores del medio que intervienen en el funcionamiento para explicar un resultado.

En cuarto lugar, es indispensable tener presente que los resultados de la actuación del sector público pueden ser evaluados más fácilmente si están estandarizados, pero no todas las actividades son apropiadas para esa estandarización. Por ejemplo, se puede estandarizar la entrega de beneficios económicos a poblaciones de bajos ingresos, de manera que se cuente con parámetros para definir quiénes deben ser conside- 
rados como pertenecientes a esta población, se pueden construir bases de datos para ubicarlas y cálculos para conocer qué cantidad de beneficios deben ser entregados. Pero existen otros servicios que son más difíciles de cuantificar porque no son cuantitativos sino cualitativos, por ejemplo la atención de pacientes en el servicio de salud donde cada caso es diferente, o bien la labor de consejerías como las que se realizan en el Patronato Nacional de la Infancia, en la Caja Costarricense de Seguro Social o en el Ministerio de Trabajo. Este segundo tipo de servicios requiere evaluaciones orientadas a medir el impacto que están generando en la sociedad, a través de los años.

También podrían realizarse estudios de costos para medir si la eficiencia se ajusta a ciertos parámetros, pero no siempre es posible, por lo que, como se indica anteriormente, en muchos casos sería necesario elaborar estudios de impacto para valorar los cambios que algunos programas han provocado en la sociedad.

Claro está que aun en los casos en los que los objetivos sean fácilmente medibles, si ellos han sido especificados muy vagamente, será dificultoso saber qué medir porque sus resultados no tienen una proposición inicial para compararlos y si bien al final pueden ser evaluados, sería un trabajo que requiere de grandes esfuerzos y recursos.

En quinto lugar, otra de las grandes complejidades del sector público se refiere al hecho de que existen contradicciones en cuanto a la rapidez con que se necesitan los resultados y las reglamentaciones que le exigen ir paso a paso y bajo la vigilancia de los entes contralores.

Es decir, se requiere mayor eficiencia, brindar los servicios a tiempo, lo cual, tal y como lo indica Pullit (2003), solo es posible si se les da una serie de autonomías para que estos actúen de acuerdo con las necesidades, ya que para lograrlo se requiere de creatividad, y cierta libertad con el fin de dar soluciones en el momento oportuno. En otras palabras, "se necesita dejar a los administradores administrar" (Pullit, 2003, p. 83).

Sin embargo, existe todo un marco legal que restringe y fiscaliza cada uno de los pasos de los gerentes públicos, a lo que se puede agregar que, cada vez que un acto de corrupción es descubierto, se tiende a aumentar el control mediante un marco legal más estricto, con lo cual puede ser que al final no desaparezcan dichos actos pero se entorpece la labor de la administración pública. Es decir, se endurece el marco legal en lugar de evaluar si lo adecuado es mejorar los procedimientos de control.

Es necesario mencionar aquí el tratamiento que se da a las ineficiencias con que se brindan los servicios del sector público. Por ejemplo, en el caso costarricense, frente a este fenómeno se ha hecho un llamado a realizar un traslado de funciones del sector público al privado, con lo cual vemos en los últimos años una serie de adjudicaciones de carreteras, aeropuertos y otras actividades. No se sabe aún si este fenómeno en realidad traerá los beneficios en cuanto a la eficiencia y eficacia que se ha prometido, puesto que no existe un verdadero análisis de esas adjudicaciones. Además, no se sabe si tales servicios pueden ser fácilmente controlables por la administración pública una vez que han sido adjudicados, o al final este control será más costoso que el que se daría si es ejecutado por la propia institucionalidad del sector público. Tampoco se conoce si esas adjudicaciones se pueden modificar o corregir para satisfacer las nuevas necesidades de la ciudadanía que vayan surgiendo en el tiempo. En síntesis, se desconoce si en realidad simplifican el trabajo de la administración pública o lo complican aún más. Tampoco se cuenta con un panorama claro sobre los costos de esta nueva forma de operar los servicios públicos.

En sexto lugar, debemos analizar el contexto inestable en que se desenvuelve el gerente pú- 
blico de nivel medio, hecho que no puede dejarse de lado a la hora de evaluar los resultados de los gerentes de más alto nivel. Se refiere aquí al hecho de que con cada cambio de gobierno a los mandos medios se les indica que deben volver la página de las labores que han venido llevando a cabo para dedicarse a los distintos programas que el nuevo gobierno requiere con urgencia, y en medio de esto se les pide que deben ser más transparentes en sus acciones, que sus acciones deben ser más medibles, más auditables, que no tomen decisiones importantes que afecten a grupos particulares, que deben tomar en cuenta a los organismos de control, a las autoridades sectoriales, a los ciudadanos interesados. Por lo tanto, no se puede evaluar con justicia su actuación sin tomar en cuenta que el contexto en el que se desenvuelven condiciona su eficiencia y que debe ser tarea del máximo jerarca evitar esas turbulencias apoyándolos para la ejecución de las nuevas funciones.

Sumado a lo anterior tenemos la otra cara de la moneda, siendo necesario anotar que para evaluar al sector público se debe tener presente que la responsabilidad es un asunto de gran complejidad. Por ejemplo, los gerentes, que son nombrados en cada nuevo gobierno, llegan a administrar organizaciones cuyos empleados se encuentran contratados por sistemas que los hacen prácticamente inamovibles. Esos empleados tienen sus propias costumbres, su ideología y sus formas de hacer el trabajo y en muchos casos están amparados por fuertes organizaciones sindicales que los respaldan. Frente a todos ellos se encuentra ese gerente de alto nivel, a quien se le pide respuestas que al final están en manos de esos empleados que no conoce, no ha contratado y no sabe cuál es su rendimiento. Más aun, muchas veces el gerente no ha podido contratar ni aun a sus colaboradores más cercanos, porque, existen compromisos políticos que propician que sean otros los que deciden quiénes son sus segundos al mando. Esos segundos, en muchos casos, no conocen el sector y no tienen la capacitación necesaria para ejecutar los programas con los cuales está comprometido el máximo jerarca. Es por esta razón que en medio de todo, los mandos medios cobran una gran importancia puesto que son quienes tienen la información y el conocimiento del sector, razón por la cual en muchas ocasiones la primera tarea del máximo jerarca consiste en ganarse la confianza de ellos para lograr el trabajo y la información que necesita.

A partir de este panorama, la cuestión de responsabilidad de los gerentes públicos toma matices muy complejos, ya que como máximo representante de la Institución es quien asume la responsabilidad frente a la opinión pública que desconoce las razones de por qué no se toman las decisiones de manera expedita.

En sétimo lugar debe tenerse presente que en cualquier administración pública las decisiones no se toman de manera individual, siempre hay varias oficinas y distintos niveles jerárquicos involucrados. Tal y como señala Bañón (2003), lo complejo es que las políticas públicas las formulan y las ejecutan varias personas y cada una de ellas filtra la realidad a través de sus propios valores, de su capacidad técnica, de sus intereses, de sus circunstanciales, de su grado de información, a lo cual debe agregarse hasta de su ideología política.

Es interesante tomar en cuenta aquí que los nuevos enfoques sobre el funcionamiento de la administración pública, tal como los de la Nueva Gestión Pública (Olías de Lima, 2001) sugieren una mayor participación del público en el accionar del sector público. Esto que a primera vista pareciera algo novedoso, incrementa las complejidades administrativas sin asegurar los beneficios de dicha participación. Debe tenerse presente que podría ser una práctica poco conveniente si se lleva al extremo por cuanto, tomar decisiones por consenso o al menos por mayo- 
ría al participar la comunidad requiere tiempo para ponerse de acuerdo, aprender a trabajar en equipo, capacitar a todos los actores, entre otros. Es decir, factores que aumentan la complejidad y retrasan la ejecución (Pullit, 2003).

Por lo tanto, evaluar los gerentes públicos y obligarlos a dar cuentas de sus actos es necesario, pero sin dejar de lado el contexto en el que se desenvuelven. No se trata aquí de hacer ver que todas estas complejidades no permiten una evaluación justa, lo que no se puede obviar es el contexto porque entonces la evaluación se convertiría en un arma peligrosa para el gerente, para la institución y para la buena marcha del sector.

Tampoco se trata de presentar un panorama trágico del sector, mucho de este accionar se puede cambiar y se verán mejoras pero lo que se trata aquí es de hacer ver que la Administración Pública siempre tendrá sus particularidades.

\section{Efectos de la evaluación en la opinión pública}

De esta manera surgen preguntas como: ¿el informante dispone de suficiente información para emitir una noticia?, ¿se busca solamente una noticia impactante?, ¿el receptor realmente tiene independencia como para ofrecer un juicio justo?, ¿la ciudadanía tiene competencia para entender las complejidades de la administración pública?

Algunos autores como Pullit (2003) sostienen que se obtienen mejores resultados en instituciones que se fijan indicadores pero que no están expuestos al ojo público que aquellos que sí lo están, porque los primeros pueden atender y corregirse ante los reclamos, mientras que los segundos deben justificarse y apegarse a la defensa de sus resultados; a pesar de todo, la realidad es que la evaluación es necesaria.
En realidad, como el Sector Público requiere informar con transparencia, lo que debe tenerse es el cuidado de que exista un aprendizaje conjunto en donde gerentes comprendan y se involucren en sus instituciones de manera que puedan explicar con toda claridad el avance de sus programas, mientras que los evaluadores tengan la obligación de estudiar a profundidad estas situaciones de manera que más que buscar la noticia rápida, dediquen tiempo al análisis del sector para cuestionar los resultados.

Por otra parte, puede suceder que con un desconocimiento total de estos procesos se realicen juicios de valor que pongan en peligro la credibilidad de programas muy importantes para el país y para la calidad de vida de muchos ciudadanos. Un ejemplo hipotético -para su mejor comprensión- sería la evaluación de un nuevo programa para triplicar el número de becas escolares a nivel nacional. Esto podría parecer tan fácil como construir una lista de niños a los cuales debe entregarse cierta cantidad de dinero. De esta manera puede ser que en el mismo semestre en que se trabajaba en la elaboración de una base de datos confiable de los niños a nivel nacional, los medios de comunicación se encuentren evaluando la ineficiencia del programa por no entregar las becas a tiempo, el impacto del programa de becas en la deserción escolar, las compras que hacen los estudiantes con el dinero, la falta de vigilancia del ministerio encargado sobre el uso del dinero, entre otros.

De nuevo, la pregunta aquí, es con cuál marco de análisis se elaboran juicios, cómo informa al público sobre el trabajo de la administración pública y, tal y como se dijo anteriormente, qué efectos tiene en el ciudadano al ser informado sobre la manera ineficiente con que se entrega un servicio.

Una evaluación negativa puede generar desmotivación a nivel nacional, desgano para pagar impuestos y otras implicaciones. Pullit (2003) ex- 
plica lo anterior con un ejemplo sobre la medida que se hace a las escuelas públicas en Inglaterra por medio de un test SAT (Scholastic Aptitude Test) que es un examen que se aplica a ciertos estudiantes para valorar los conocimientos adquiridos, pero cuyo resultado sirve para evaluar a todos los demás estudiantes y a toda la escuela.

Señala Pullit (2003) que si la escuela está por debajo del promedio nacional, los padres tienden a trasladar a sus hijos, lo cual trae grandes problemas a la escuela como dificultades para conseguir presupuesto y dificultades para conseguir buenos educadores. También conduce a cambios económicos en la comunidad al tener menos escolares en el área y habitantes buscando residencias en otros lugares para trasladar a sus hijos de centro educativo. Casi nadie sabe, dice este autor, qué es el SAT, pero afecta enormemente a la escuela y a la comunidad, de manera que según su criterio, el SAT puede no ser malo, pero el uso que se le da a la información que se obtiene de este sí que lo es.

En el mismo sentido, puede darse el caso de que evaluaciones poco científicas se encarguen de extender la creencia que toda actividad del sector público es ineficiente mientras que toda actividad del sector privado es por naturaleza eficiente, aunque estudios empíricos han demostrado que no es siempre cierto que la empresa privada obtiene mejores resultados que la pública, lo cual ha quedado demostrado en algunos casos cuando se privatizan actividades públicas (Jackson, 2001 citado por Pollitt y Bouckaert, 2011).

Indica Pollitt y Bouckaert (2011) que la razón de esta confusión obedece a que se confunde la eficiencia con la eficacia. Mientras que eficiencia se refiere a la diferencia entre recursos utilizados versus bienes o servicios producidos; por ejemplo, el número de lecciones impartidas en una escuela pública versus cantidad de millones invertidos en profesores. La eficacia se refiere al grado en que una organización ejecuta sus ob- jetivos, por ejemplo, si reciben los niños el número de lecciones previamente programadas o bien, si reciben en general los beneficios sociales acordados.

Dice este autor que no se toma en cuenta que es posible ser eficiente sin ser efectivo y viceversa. En este sentido las empresas privadas tienden siempre a trabajar con la menor cantidad de recursos aunque puede no ser tan efectiva, ya que atiende la cantidad de personas que conviene a sus mayores beneficios sin importar si dejan parte de la población sin atender. Mientras el sector público no puede obtener esos grados de eficiencia porque debe ser eficaz, es decir, debe atender a todos sin distinción.

Se hace necesario agregar aquí que otro de los análisis que conducen a confusión es la correcta definición entre eficacia y efectividad. De esta manera la eficacia con que se cumplen los objetivos, -impartir el número de lecciones previamente programadas-, no tiene que ver con la verdadera efectividad que se obtiene cuando los alumnos realmente aprenden. De la misma manera la eficacia que se obtiene al brindar el número de ayudas programadas podría no reflejarse en la efectividad de lograr un verdadero impacto en la calidad de vida de los beneficiarios.

Para ejemplificar aun más las consecuencias de estos juicios erróneos, vemos cómo muchas veces se afirma que las actividades del sector público son ineficientes sin tener una idea de sus costos, causando preocupación a nivel nacional, cuando lo que podría estar sucediendo es que no pueden entregar la cantidad de servicios que le demandan porque se carece de recursos y lo que realmente está sucediendo es que son ineficaces pero no ineficientes.

De la misma manera, podrían darse la idea de que son muy eficientes cuando realmente lo que está sucediendo es que se brindan los servicios con cierto grado de eficacia pero a un alto costo. Cabe aquí la pregunta para algunas de las 
concesiones que tiene el estado con el sector privado: ¿son realmente eficientes?

\section{Criterios para juzgar la responsabilidad de los gerentes públicos}

Aun dentro del contexto anterior, lo primero que este apartado se plantea es que la evaluación de la administración pública y de los gerentes públicos, es no solo posible sino también necesaria.

Sin tratar de ser exhaustivo, porque no sería posible, este apartado pretende únicamente anotar algunas herramientas importantes para evaluar los gerentes y sus actuaciones de manera objetiva, porque el ciudadano tiene el derecho a estar informado de manera transparente sobre la utilización que se da a sus recursos y los gerentes tienen la obligación de informar sobre el avance de los programas que dirigen, por lo que deben ser juzgados por ello con imparcialidad.

Las evaluaciones son posibles para cualquiera que posea el conocimiento sobre el funcionamiento del sector público. Así por ejemplo, entre otros, se puede colocar toda la atención en juzgar los resultados de las instituciones públicas con base en: los indicadores planteados, se puede analizar si los indicadores están correctamente definidos, si son concretos, o bien si sirven como medida de las actuaciones específicas que se están estudiando.

Además, el debate puede orientarse a determinar porqué no existen tales indicadores y cómo están midiendo los gerentes sus propios resultados.

Otro de los aspectos que debe ser objeto de evaluación es el proceso de definición de las políticas públicas. Según Bañón (1997), puede evaluarse desde el proceso de definición hasta la ejecución de políticas públicas, para lo cual sería conveniente centrarse en:
- Los aspectos externos a la administración pública, preocupándose por el problema a tratar, sus dimensiones, su gravedad, el número de personas a las que afecta, entre otros.

- La implicación y comportamiento de los actores sociales críticos con intereses en el proceso de la política.

- Los objetivos y las metas fijadas, interesándose por conocer la adecuación entre las previsiones y los logros.

- Los medios e instrumentos utilizados en la ejecución -recursos humanos, financieros, organizativos y tecnológicos- para desarrollar la política.

Existen otros investigadores como Pullit (2003), quien también elabora una lista de aspectos que pueden ser evaluados:

- La manera imprecisa con que se definieron los objetivos de un programa

- El cumplimiento de los objetivos

- El costo

- Los procedimientos para ejecutarlos

- Los datos que se le ofrecen si son o no correctos o imprecisos

- El impacto que el programa tiene en el contexto

- El tiempo empleado en el desarrollo del programa, según los cálculos iniciales y según los atrasos cuya responsabilidad puede estar diluida en una serie de instituciones y unidades difíciles de distinguir, pero que se pueden investigar. 
Además Bañón (2003) establece que al evaluar las políticas públicas, se podrían utilizar metodologías como "investigación para la acción", herramienta con la cual se puede ayudar al público a responder preguntas como: ¿debería intervenir el gobierno para solucionar un problema concreto?; ¿cómo se debe orientar esa intervención?; ¿cuánto tiempo será necesario actuar sobre él?; ¿qué costes acompañarán a las acciones emprendidas?; ¿quién se beneficiará de la intervención y quién resultará perjudicado por ella?; ¿serán o no satisfactorios los resultados de la intervención?

Por otra parte, al igual que la evaluación por indicadores planteada anteriormente, se pueden realizar estudios de impacto en donde se muestren los cambios o resultados que se han dado en la sociedad a través del tiempo, como producto del desarrollo de uno o varios programas públicos.

De la misma manera pueden determinarse los costos incurridos en el cumplimiento de los distintos proyectos o programas, el número de ciudadanos a los que se les brinda el servicio, las competencias que dispone un gerente público para realizar su labor y muchos otros aspectos, que es necesario someter a escrutinio público.

Lo conveniente sería desarrollar una cultura evaluativa que permita al país contar con debates sobre todos estos elementos relacionados con los servicios públicos.

Estas evaluaciones son imperiosas porque de esta manera se obliga a los gerentes públicos a dar verdaderas cuentas sobre sus actuaciones y porque además es necesario desmotivar aquellos quienes no cuentan con verdaderas capacidades para desempeñar estos puestos.

Finalmente, es importante tomar en cuenta que la evaluación dada sobre bases poco científicas deja espacio a que imperen los intereses políticos particulares antes que las verdaderas necesi- dades de la ciudadanía. Así por ejemplo, puede ser que si no existen parámetros con los cuales medir la ejecución, en su lugar lo que domine sea el buen discurso de algunos gerentes que convencen a ciertos miembros de la prensa o a los mismos usuarios, lo que puede hacer aparecer la institución mejor calificada de lo que realmente es.

En el mismo sentido pueden darse juicios injustos que predispongan a la ciudadanía a aceptar decisiones que no les convienen, tal es el caso de concesionar actividades que son estratégicas para el país bajo el argumento que no se pueden administrar bien con las reglas de la institucionalidad pública.

\section{CONCLUSIONES}

Como ha quedado demostrado, colocar a los gerentes públicos y las instituciones en el centro de la discusión pública es un trabajo de enorme responsabilidad que requiere gran conocimiento sobre el funcionamiento del sector público.

Estudiar a profundidad el sector público, es la primera condición para realizar cualquier evaluación, máxime si se trata de informar a la ciudadanía sobre el acontecer en este sector. Parte de ese conocimiento radica en tener claro que la ejecución de los programas nacionales, por las características particulares que presentan, toma tiempo, la mayoría de las veces años, por lo que las evaluaciones o juicios públicos sobre sus resultados no pueden ser llevadas a cabo tan pronto como estos sean emprendidos. En su lugar, es necesario dar a conocer a los ciudadanos, entre otros aspectos, cómo avanza el diseño y la construcción de los distintos proyectos, cuál es el cronograma con que se ejecutan las obras, cuáles recursos se están empleando, para lo cual se requiere contar con conocimiento sobre el proceso que se ejecuta. 
Desde esta perspectiva tampoco es suficiente con trasladar la producción de bienes y servicios al sector privado. Si imaginamos que el gobierno dejara de existir y trasladamos todas las funciones al sector privado, podríamos arribar a la conclusión de que este último comience a sufrir los mismos problemas que tiene el primero al introducir en él casi todas las anteriores complejidades.

Tal y como ha sido explicado, la importancia de efectuar este análisis radica en que la evaluación, sobre todo cuando es llevada a juicio mediático, tiene consecuencias para el país. Algunas de ellas buenas cuando se trata de evaluaciones que permiten retroalimentar las funciones, mientras que otras pueden crear caos, confusión, desmotivación en los ciudadanos, en los funcionarios públicos y es probable que conduzcan a diseñar políticas y a tomar decisiones equivocadas.

De la misma manera, leer un conjunto de números que demuestran resultados de una institución pública puede ser más cercano a la realidad en ciertos campos que en otros.

Los enfoques conocidos como la Nueva Administración Pública y su propuesta sobre indicadores para evaluar la gerencia o las instituciones en general, pueden ser muy útiles en ciertos campos, pero pueden generar desinformación en ciertos niveles del proceso de ejecución.

Como fue dicho anteriormente, si bien las reformas que se llevan a cabo en el sector público tienen distintas etapas -planificación, implementación y resultados-, si no se cuenta con un verdadero conocimiento sobre el sector público es posible que se evalúe lo que sucede en las primeras, y menos en las siguientes, tendiéndose a no dar seguimiento a lo que acontece en ciertos campos hasta que sucede una crisis o la ciudadanía reclame la ausencia de servicios que no le son entregados.
Finalmente, a la prensa le corresponde el papel de evaluador objetivo y tiene la obligación de prepararse para examinar el sector público y someter a debate hechos basados en un análisis lo más científico posible. No se trata aquí de soslayar el hecho de que la evaluación siempre tiene un contenido político y subjetivo, pero lo que se trata es de no olvidar que un sector tan complejo requiere un conocimiento profundo y que existen herramientas científicas que pueden ser utilizadas en lugar de los simples juicios subjetivos.

\section{REFERENCIAS}

Acosta, M. (2012). Contralora General de la República. El control de la gestión pública en Costa Rica. Manuscrito inédito, Lección inaugural de segundo cuatrimestre de la Escuela de Ciencias de la Administración. UNED, Costa Rica. Recuperado de http://www.ondauned. com/transmision.php?ou=233.

Bañón R. y Carrillo, E. (1997). La Nueva Administración Pública. Alianza Universidad. Madrid. Recuperado de http://168.243.1.4/mcp/media/archivo/f98099_tamayosaezelanalisisdelaspoliticaspublicas.pdf

Castillo, A. (2012). El perfil del gerente público. Revista Nacional de Administración, 3(2)

Dirección de Cultura del Ministerio de Cultura de Costa Rica. (2010). Primera Encuesta Nacional de Hábitos y Prácticas Culturales 2010 (ENHPC). UNESCO. San José, Costa Rica.

Maingón T. (1991). Proceso De Privatización En Los Servicios De Salud En América Latina y El Caribe: Estudio De Casos. PROLAP, UNAM México. Recuperado de http://terceridad.net/PyPS/Por_temas/11_Analis_PS/Apoyo_1/ Proceso\%20de\%20privatizaci\%F3n\%20en\%20los\%20 servicios\%20de\%20salud\%20en\%20Am\%E9rica\%20 Latina\%20y\%20el\%20Caribe\%20pp.48.pdf

Molina, N. y Chowla, P. (2008). El Banco Mundial y la privatización del agua: El dinero público se va por el desagüe. Recuperado de http://www.brettonwoodsproject. org/es/2008/10/art-562616/

Olías de Lima, B. (2001). La Nueva Gestión Pública. Prentice Hall. Madrid 
RNA Revista Nacional de Administración

Periódico Digital CRHOY (9 de octubre 2013). Autopistas del sol aún le falta cobrar el 67\% de ganancias por peajes. Recuperado de: http://www.crhoy.com/autopistasdel-sol-aun-le -falta-cobrar-el-67-de-ganancias-porpeajes/

Pollitt C. y Geert B. (2011). Public Management Reform: A Comparative Analysis - New Public Management, Governance and the Neo-Weberian State. Oxford University Press

Porter, R. (2010). Influencia de los juicios paralelos por los medios de comunicación colectiva en el proceso penal. Observatorio Judicial. Poder Judicial, 17 de noviembre de 2010, edición 112. República de Costa Rica.

Pullit, C. (2003). The essential public manager. Open University Press. Londres: Mc Graw Hill.

Robbins, S. y Coulter, M. (2011) Administración. Pearson. México

Schröder, P. (2009). Nueva Gestión Pública: Aportes para el buen gobierno. Fundación Friedrich Naumann, Oficina Regional América Latina. Recuperado de www.la.fnstfreiheit.org/uploads/.../Schroeder_NGP

Zurcher, J. (2014). ¿Privatización? Periódico la Nación, Costa Rica, 14 de enero 2014.

Recibido: 2 de junio de 2013 Aceptado: $1^{\circ}$ de noviembre de 2013 